\section{A BUSINESS MODEL TO INSPIRE AND MOTIVATE}

Committed to excellence, the British Academy of Cosmetic Dentistry's Study Club events are great opportunities to benefit from the experience and knowledge of speakers who are leaders in their field.

The BACD Bristol Study Club on Tuesday 19th October 2010 is entitled 'The Million Pound Dental Practice'. The event will show examples of practices that are able to create a positive working environment, offer great earnings and a career pathway to the team, develop first class customer service and give patients the best dentistry available, amongst many benefits. With his wealth of experience in coaching and mentoring dental practices, Mr Chris Barrow will show how to develop a business model that is sure to inspire and motivate.

Reader response number 58

\title{
ELIMINATE TIRING AND STRENUOUS MANUAL PROCEDURES
}

Every dentist needs a fantastic assistant and the new iSD900 cordless prosthodontic screwdriver from NSK certainly fits the bill for implant dentists. The iSD900 can be used with all major implant systems, guaranteeing precise torque control whichever implant system you use.

The iSD900 cordless screwdriver features NSK's unique Torque Calibration

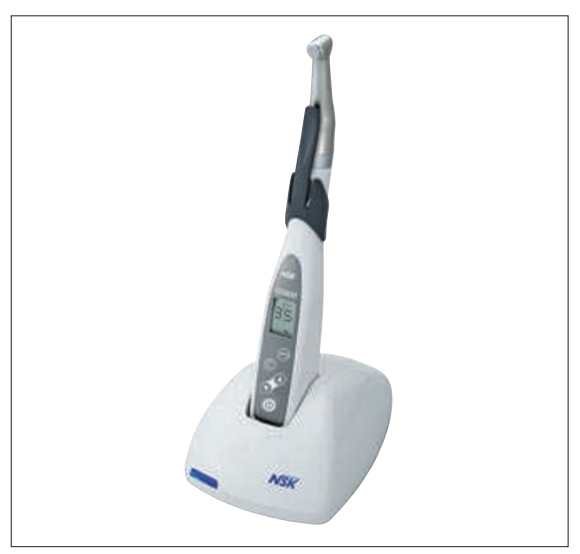

System (TCS) which ensures that the correct torque is applied at all times. This helps the clinician to screw and unscrew abutments reliably and without risk, eliminating tiring and strenuous manual procedures.

The iSD900's LCD digital display clearly shows torque, speed, reverse rotation, battery condition and calibration mode settings. With precise torque from $10-40 \mathrm{Ncm}$ available in 1 or $5 \mathrm{Ncm}$ increments and speeds of 15, 20 or 25 rotations per minute, the screwdriver has settings to suit every individual case. The iSD900's advanced battery and charger technology includes a fast charge of only 90 minutes, meaning this assistant is always ready for work.

The iSD900 cordless prosthodontic screwdriver from NSK is available exclusively from Straumann UK.

Reader response number 59

\section{RAPID OBTURATION}

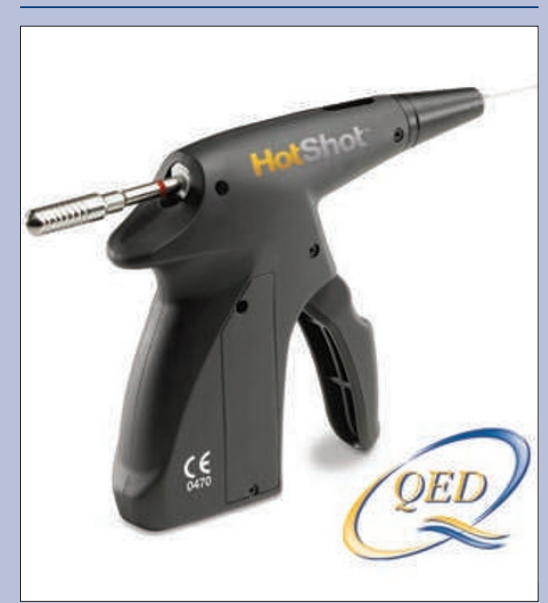

QED are the sole UK distributors for the new HotShot ${ }^{\mathrm{TM}}$ Cordless Backfill Obturation Delivery Device.

The HotShot enables fast and easy delivery of warm obturation material for the endodontic backfill technique, facilitating rapid obturation of even the most difficult root canals more completely, in less time, and with less patient discomfort.

The HotShot is cordless, compact and easy to handle. It features variable temperature settings which allow practitioners to control the flow characteristics of the material; a rechargeable lithium ion battery which will support hours of use from a single charge; and a choice of needle size of either $23 \mathrm{~g}$ or $25 \mathrm{~g}$ with a $360^{\circ}$ swivel for improved access.

Reader response number 60

\section{CONE BEAM FOR IMPLANTOLOGISTS}

The Galileos Compact from Sident Dental Systems offers a convenient entry to the world of 3D cone beam digital diagnostics, with the ability upgrade for expansion. It offers unparalleled functionality in diagnosis, planning and treatment associated with the Galileos Comfort, which also includes a CEPH facility.

Galileos 3D cone beam digital X-ray systems offer practices the power of integrated diagnostics and treatment planning via a single imaging system. It enables them to reduce risks, plan surgical interventions, co-ordinate treatment planning with colleagues and explain treatment to patients with even greater clarity, certainty and ease.

With their innovative cone beam technology, Galileos systems can calculate a large volume 3D image set (over 200 exposures) in a single low-dose scan lasting 15 seconds or less. This 3D image set is then processed and displayed using their integrated GALAXIS 3D software.

The Galileos Compact presents its 3D images in perfect image quality and facilitates easy navigation and diagnosis in traditional PAN and TSA presentations. The Galileos Comfort offers CEPH views too. Both are all-in-one diagnosis and planning systems which offer a very short, low dose scanning cycle for optimum speed and efficiency.

Reader response number 61

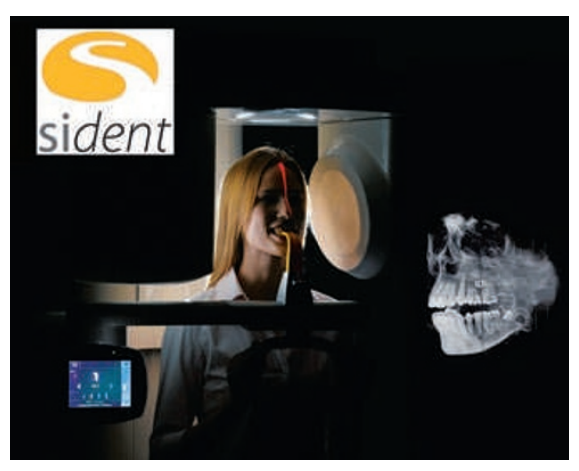

